\title{
Anxiety-related symptomatology in young children on the autism spectrum
}

\section{Short Title: Anxiety in young autistic children}

Deb Keen ${ }^{1,2}$. Dawn Adams ${ }^{1,2}$. Kate Simpson ${ }^{1,2}$. Jacquiline den Houting ${ }^{1,2}$. Jacqueline Roberts ${ }^{1,2}$

${ }^{1}$ Autism Centre of Excellence, School of Educational and Professional Studies, Griffith University, Australia

${ }^{2}$ Cooperative Research Centre for Living with Autism (Autism CRC), Long Pocket, Brisbane, Queensland, Australia.

\section{Corresponding Author:}

Prof. Deb Keen, Autism Centre of Excellence, School of Education and Professional Studies, Griffith University, Mt Gravatt, Brisbane, QLD 4122, Australia Email: D.Keen@Griffith.Edu.Au 


\section{Acknowledgements}

We are grateful to the children with autism and their families for giving their time to support this research study. The authors acknowledge the work of the LASA team members: Susan Bruck, Trevor Clark, Sandra Devaraj, Robyn Garland, Honey Heussler, Antoinette Hodge, Patricia Howlin, Jessica Paynter, Natalie Silove, David Trembath, Madonna Tucker, Marleen Westerveld, Katrina Williams. The authors also acknowledge the financial support of the Cooperative Research Centre for Living

with Autism (Autism CRC), established and supported under the Australian Government’s Cooperative Research Centres Program.

\section{Funding statement and declaration of conflicting interests}

The authors have no conflict of interest with respect to this publication.

The authors acknowledge the financial support of the Cooperative Research Centre for Living with Autism (Autism CRC), established and supported under the Australian Government's Cooperative Research Centres Program 


\title{
Anxiety-related symptomatology in young children on the autism spectrum
}

\author{
Short Title: Anxiety in young autistic children
}

\begin{abstract}
Anxiety symptomatology is frequently reported in autistic children and the prevalence of anxiety disorder is estimated at around $40 \%$. However, most studies have focused upon children age 8 or over, so little is known about early signs of anxiety in younger children with autism. This study sought to describe anxiety-related symptomatology in 95 5-6 year old autistic children using the Anxiety Scale for Children with Autism Spectrum Disorder (ASC-ASD). Wide variability was found in levels of symptomatology with the most frequently reported items within the 'uncertainty' subscale and the least frequently reported items in the 'anxious arousal' subscale. Comparisons of those with scores $</>70$ on adaptive behaviour suggests some influence of ability on presentation of anxiety-related symptomatology.

Keywords
\end{abstract}

Anxiety, autism spectrum disorder, autistic children, parent report 


\section{Anxiety-related symptomatology in}

\section{young children on the autism spectrum}

It has been recognised for some time that autistic children ${ }^{1}$ are at increased risk of anxiety, with prevalence of anxiety disorders estimated at around 40\% (van Steensel et al., 2011). This compares to an estimated prevalence in the general population of approximately 7 to $15 \%$ (Biedel and Turner, 2005), where anxiety disorders frequently manifest prior to the age of 16 years, and are persistent over time and across the lifespan (Beesdo et al., 2009). In the preschool years, research has identified common anxiety subtypes in the general population. Spence and colleagues (2001) examined anxiety symptoms in preschoolers with typical development aged 2.5 to 6.5 years using exploratory and then confirmatory factor analyses. They found a five-correlated-factor model of anxiety consisting of social phobia, separation anxiety, generalized anxiety, obsessive-compulsive disorder and fears of physical injury. A more recent study found that generalized anxiety disorder, separation anxiety disorder, and social phobia were common among preschool-aged children with typical development attending pediatric primary care (Franz et al., 2013). While these studies and others investigating anxiety in preschoolers with typical development has led to increased understanding and informed early intervention and prevention efforts, research specifically on anxiety symptoms in autistic preschoolers has been somewhat overlooked.

\footnotetext{
${ }^{1}$ This terminology has been adopted based on guidelines provided by Autism indicating a preference by autistic adults for the term 'autistic person'
} 
It does appear that anxiety symptoms manifest early in the life of a child on the autism spectrum, and that age is likely to be a factor in relation to type and severity of anxiety across the lifespan. In particular, anxiety symptoms appear to be more evident in older versus younger autistic children (Magiati et al., 2015). It has also been suggested that there may be a relationship between age and type of anxiety symptomatology. Specifically, overall anxiety and generalized anxiety disorder rates were found to be higher in older children, while separation anxiety and obsessivecompulsive disorder (OCD) were more common in younger children (van Steensel et al., 2011). It is important to note, however, that research to date has predominantly focused on anxiety in school-aged autistic children and youth with few samples including preschool children. This is evident in a meta-analysis of anxiety disorders in children on the autism spectrum conducted by van Steensel et al. (2011). Their metaanalysis included a total of 31 studies and of these, only two had samples with children who had a mean age of less than 6 years.

One of these studies, conducted by Gadow et al. (2004), involved a sample of 182 children with PDD and a mean age of 4.2 years (SD 0.8). Anxiety was measured using the Early Childhood Inventory-4 (ECI-4) (Gadow and Sparafkin, 1997; Gadow and Sparafkin, 2000), a parent/teacher report measure referenced to the DSM-IV. In comparison to a non-PDD control group, the PDD group received higher severity scores on general anxiety disorder and social phobia, but not on separation anxiety. A second study, conducted by Davis et al. (2011), investigated anxiety-related symptoms across the lifespan, grouping participants as toddlers (17-36 months), children (3-16 years), and adults (20-65 years). Participants with autism were recruited via early intervention services, schools, outpatient clinics and community organizations. Confirmation of diagnosis procedures varied according to the age and 
recruitment. The authors reported that anxiety in their autistic participants increased from toddlerhood to childhood, decreased from childhood to young adulthood, and then increased again from young adulthood into older adulthood (over 49 years). Findings on young autistic children in these studies suggest the need for further research to clarify the possible relationships between age and the frequency and type of anxiety symptoms that present in this age group. Increasing our knowledge and understanding of anxiety symptomatology in early childhood is important, as it can inform intervention efforts aimed at preventing later anxiety disorders. Research has shown that early intervention with a non-ASD preschool population can provide protection from development of anxiety disorders in middle childhood (Rapee, 2013) but as yet there is no evidence of whether autistic children would benefit from early intervention in this way. Having more accurate information about the presentation of anxiety symptoms in preschoolers can help to inform prevention and intervention efforts.

One of the difficulties in exploring anxiety symptoms in autistic preschoolers is the lack of appropriate anxiety measures validated with this age group. Further, there has been growing debate in the literature over the past decade about the suitability of standard anxiety measures for use with autistic children. Underpinning this debate is that differentiation of autism symptoms and anxiety symptoms is difficult (Rodgers et al., 2016b). Repetitive behaviours and interests can overlap with obsessive behaviours associated with obsessive compulsive disorder. Social anxiety may be difficult to distinguish from socialization difficulties related to the characteristics of autism. Kerns et al. (2014) have noted that presentations of anxiety in autism can be atypical, while others have stated that autistic children often don't display age-typical symptoms of anxiety (White et al., 2009). It could be said that 
“...the field is hampered by lack of a definitive conceptualization of anxiety in ASD, and the means to capture features of anxiety as a clinical disorder separate from ASD” (p12, Wigham and McConachie, 2014).

In response to this growing concern about anxiety measurement in autism, Rodgers et al. (2016b) recently published the Anxiety Scale for Children with Autism Spectrum Disorder (ASC-ASD). The ASC-ASD is a 24 item scale (self and parent report) comprised of four subscales: Performance Anxiety; Uncertainty; Anxious Arousal; and Separation Anxiety. It was designed and tested with children aged 8 to 16 years. Items were derived based on the Revised Child Anxiety and Depression Scale that has been used widely with typically developing children. The ASC-ASD has been shown to have good reliability, validity and internal consistency. It does not claim to be a diagnostic instrument, but a way to document and describe the symptoms of anxiety for individuals with autism. While the psychometric properties of the ASC-ASD are promising, validation has been limited, as the tool was only recently published. This measure is, however, the only available autism-specific anxiety measure currently available to explore anxiety-related symptoms in autistic individuals.

The aim of the current study was to investigate the frequency and range of anxiety-related symptomatology in a cohort of 5-6 year old autistic children. Given the absence of anxiety measures suited for use with this age group, the ASC-ASD was selected as the most appropriate tool to use to explore anxiety-related symptoms and to examine any associations with levels of adaptive ability. 


\section{Method}

This study used data collected through the Longitudinal Study of Australian Students with Autism (LASA). The LASA was designed to investigate educational and participation trajectories and outcomes for Australian autistic students over a six year period. The methodology for this study is briefly described here with further details available in Roberts et al. (in press). Ethical approval for this study was obtained from participating universities and health authorities.

Parents of autistic children aged 4-5 years $(n=132)$ and 9-10 years $(n=134)$ living in Australia were recruited in 2015 into the LASA through service providers, support groups and electronic media. To date, data have been collected from parents at two of the six annual time points via an online questionnaire consisting of developmental and behavioural assessments together with a range of demographic questions.

\section{Participants}

Ninety-five parents of autistic children aged 5-6 years at the second time point (T2) of the LASA were selected for this study. This time point was selected as it marked the introduction of the then newly published ASC-ASD measure in the longitudinal study. Only participants who had complete datasets from the anxiety measure and who scored above the autism cut-off for young children on the Social Communication Questionnaire (described below) were included. A subsample of 64 of these participants had complete data from the Vineland Adaptive Behavior Scales. Demographic characteristics of the participants and children are displayed in Table 1. 
Additional diagnoses are based upon parent self-report in response to an open question asking parents to list any additional diagnoses the child had received.

Table 1. Demographic characteristics for the full sample and subsample

\begin{tabular}{|c|c|c|c|}
\hline & & \multicolumn{2}{|c|}{ Mean (SD) or $n(\%)$} \\
\hline & & \multirow{2}{*}{$\begin{array}{l}\text { Total } \\
\mathrm{N}=95\end{array}$} & \multirow{2}{*}{$\begin{array}{l}\text { Subsample } \\
\text { with VABS } \\
n=64\end{array}$} \\
\hline & & & \\
\hline \multicolumn{2}{|l|}{ Age of child (years) } & $6.1(.53)$ & $6.1(.54)$ \\
\hline \multicolumn{2}{|l|}{ Gender of child (male) } & $79(83.2 \%)$ & $52(81.3 \%)$ \\
\hline \multicolumn{2}{|l|}{ Indigenous origin } & $1(1.1 \%)$ & $1(1.6 \%)$ \\
\hline \multirow{5}{*}{$\begin{array}{l}\text { Additional diagnosis or } \\
\text { medical condition as } \\
\text { reported by parents }\end{array}$} & Any additional diagnosis & $50(52.6 \%)$ & $36(56.3 \%)$ \\
\hline & ADHD & $14(14.7 \%)$ & $9(14 \%)$ \\
\hline & Asthma & $5(5.3 \%)$ & $4(6.3 \%)$ \\
\hline & $\begin{array}{l}\text { Intellectual disability or global } \\
\text { developmental delay }\end{array}$ & $6(6.3 \%)$ & $3(4.7 \%)$ \\
\hline & Anxiety & $8(8.4 \%)$ & $6(9.4 \%)$ \\
\hline \multicolumn{2}{|c|}{ Informant education (tertiary education or above) } & $73(76.8 \%)$ & $50(78.1 \%)$ \\
\hline \multirow{3}{*}{$\begin{array}{l}\text { Informant main } \\
\text { occupation }\end{array}$} & Employed & $56(59 \%)$ & $39(60.9 \%)$ \\
\hline & Full-time carer & $31(32.6 \%)$ & $19(29.7 \%)$ \\
\hline & Studying & $6(6.4 \%)$ & $4(4.7 \%)$ \\
\hline \multirow[t]{2}{*}{ Child education setting } & $\begin{array}{l}\text { Mainstream } \\
\text { support) }\end{array}$ & $80(84.2 \%)$ & $55(85.9 \%)$ \\
\hline & Special School & $15(15.8 \%)$ & $9(14.1 \%)$ \\
\hline
\end{tabular}




\section{Measures}

The Social Communication Questionnaire (SCQ) (Rutter et al., 2007) is a parentcompleted, well-validated screening test for autism. Parents indicate the presence of social, communicative and stereotyped behaviours by responding yes or no to 40 items. Within the current study, a cut-off of 11 was used to validate the diagnosis of autism as per Eaves, Wingert, Ho and Mickelson's (2006) research with young children.

The Vineland Adaptive Behavior Scales $2^{\text {nd }}$ Edition (VABS-2) (Sparrow et al., 2005) was used to assess adaptive behaviour and information was collected on three domains: socialization, daily living skills, and communication.

The Anxiety Scale for Children with Autism Spectrum Disorder (ASC-ASD) (Rodgers et al., 2016b) was completed by parents and provides a measure of anxietyrelated symptomatology. There are 24 items from which four subscales have been derived: Performance Anxiety (five items; maximum score 15); Anxious Arousal (six items maximum score 18); Separation Anxiety (five items maximum score 15) and Uncertainty (eight items maximum score 24). Severity was rated on a 4-point scale with 0 (never) and 3 (always). The scale has good validity, reliability and internal consistency and is highly correlated with the Screen for Child Anxiety Related Emotional Disorders (SCARED) (Birmaher et al., 1999), a robust measure of anxiety in the general population. In the current study, internal consistency was identified as good to excellent for all subscales based on Cronbach’s alpha; Anxious Arousal $\alpha=.80$, Separation Anxiety $\alpha=.84$, Performance Anxiety $\alpha=.89$ and Uncertainty $\alpha=.91$.

\section{Data analysis}


To explore the profile of scores and the most and least frequently endorsed on the ASC-ASD in young children with autism, scores were reported at item and subscale level for the full sample. Pearson's correlations were calculated between the ASCASD subscale scores and SCQ total score to investigate the association between characteristics of autism and scores on the ASC-ASD. As the four subscales of the ASC-ASD have different numbers of items, subscale scores were presented as mean score per item to allow for comparisons across subscales.

ASC-ASD subscale scores were further explored for the subsample of children who have complete VABS-2 scores for all three domains (daily living skills, socialization and communication). For each domain, participants were divided into two groups based upon their standard score; 70 and below and 71 and above. Because the four anxiety subscales are moderately correlated with each other, a multivariate analysis of variance (MANOVA) was computed to compare the mean anxiety ratings on the 4 subscales between those of higher and lower ability on each of the three domains from the VABS. MANOVA was selected as it is deemed robust to violations of non-normality when ten or more participants are included in each group (Seo et al., 1995). As per Tabachnick and Fidell (2007), due to unequal sample sizes, Box’s M test for homogeneity of variance-covariance matrices was considered prior to any analyses. The alpha for Post-hoc ANOVA comparisons was Bonferroni corrected to .013 to correct for the 4 additional comparisons made following each MANOVA. All statistical analyses were undertaken on SPSS version 24.0.

\section{Results}

\section{Profile of scores on the ASC-ASD}


Total scores ranged from zero $(n=2)$ to $65(n=1)$ (maximum possible score 72 ). The ASC-ASD subscale scores ranged from the minimum to maximum for all subscales except the Anxious-Arousal subscale where the maximum score reported was 13 (maximum possible 18). Skew and Kurtosis were within the range of \pm 1 for all subscales except Anxious-Arousal, which had a slightly positive skew (1.4) and Kurtosis (2.0). The mean total score for the total sample was 22.82. For reference, the mean score in Rodgers et al. (2016b) original sample of 157 8-15 year olds was 26.19. Eight children within this sample were reported as having a diagnosed anxiety disorder, so their mean scores per item were also explored, although this must be interpreted with caution due to the reliance on parent disclosure of additional diagnoses and a small $\mathrm{n}$. The mean total score of this small subgroup was 29.78 .

The mean score per item was highest for the uncertainty scale $(\overline{\mathrm{x}}=1.36, \mathrm{SD}=$ $.76)$, then separation anxiety $(\overline{\mathrm{x}}=.92, \mathrm{SD}=.78)$, performance anxiety $(\overline{\mathrm{x}}=.82, \mathrm{SD}=$ $.82)$ and lowest for the anxious arousal subscale $(\overline{\mathrm{x}}=.54, \mathrm{SD}=.49)$. The mean scores per item for the small subgroup with a parent disclosed diagnosis of anxiety showed a similar pattern but with higher mean scores than noted for the total sample; uncertainty scale $(\overline{\mathrm{x}}=1.81, \mathrm{SD}=.66)$, separation anxiety $(\overline{\mathrm{x}}=1.13$, $\mathrm{SD}=.89)$, performance anxiety $(\overline{\mathrm{x}}=1.03, \mathrm{SD}=.97)$ anxious arousal subscale $(\overline{\mathrm{x}}=.75, \mathrm{SD}=$ $.35)$.

Table 2 shows correlation coefficients between the ASC-ASD subscales and SCQ total score for the total sample. The ASC-ASD subscales were moderately correlated with each other. The SCQ was weakly to modestly correlated with the ASC-ASD subscale scores.

Table 2. Pearson's correlations between ASC-ASD subscales and SCQ Total Score 


\begin{tabular}{lllll}
\hline & $\begin{array}{l}\text { Performance } \\
\text { Anxiety }\end{array}$ & $\begin{array}{l}\text { Anxious } \\
\text { Arousal }\end{array}$ & $\begin{array}{l}\text { Separation } \\
\text { Anxiety }\end{array}$ & Uncertainty \\
\hline Anxious Arousal & $.56^{* *}$ & - & & \\
Separation Anxiety & $.62^{* *}$ & $.63^{* *}$ & - & \\
Uncertainty & $.62^{* *}$ & $.68^{* *}$ & $.70^{* *}$ & - \\
SCQ score & .24 & $.42^{* *}$ & $.30^{*}$ & $.34^{*}$ \\
\hline
\end{tabular}

$* \mathrm{p}<.01 * * \mathrm{p}<.001$

Figure 1 presents the profile of scores for the group of 95 five and six year olds with autism at item level and highlights the range of response patterns for each question. The most frequently endorsed items were within the Uncertainty subscale, with six of the eight items occurring sometimes or more in over $80 \%$ of children and two items ("My child worries if they don't know what will happen next" and "My child always needs to be prepared before things happen”) being rated as occurring often or always in over half the sample.

Although $79 \%$ of children in the sample are noted to experience " $a$ scared feeling when there is nothing to be afraid of" at least sometimes, very few of the other Anxious Arousal symptoms were frequently endorsed, with only $7.4 \%$ of children reported to experience dizziness or faintness for no reason.

Within the Separation Anxiety subscale, almost half (44.2\%) of children were noted to worry about being away from their parents often or always. Almost twothirds $(65.2 \%)$ were noted to worry in bed at night because they do not like to be away from their parents or family at least sometimes. Comparatively few (10.6\%) parents noted that their child often or always worries that something awful will happen to someone in their family. 
Finally, almost half of the children at least sometimes experienced symptoms within the Performance anxiety subscale. Even at five or six years old, more than onethird (35.8\%) of children in the sample are often or always scared of taking tests in case they make a mistake or don't understand the question and $17.9 \%$ of the children often or always worry what other people may think of them.

++insert Figure 1 here++

\section{Comparison between those with higher and lower levels of adaptive behaviour}

The sample characteristics of the 64 children with complete data from the VABS-2 were summarized in Table 1. Of these, eleven (17.2\%) participants had scores below 70 on all three domains of the VABS-2, and 43 (67.2\%) had all scores at or above 71 on all three domains. The remaining 10 (15.3\%) had a mix of scores above and below 70.

A MANOVA was conducted using SPSS 24.0 to assess group (i.e., standard score of 70 and below or 71 and above on the domains of the VABS-2) differences on the four ASC-ASD subscales. They revealed a significant main effect of level of ability across all three domains (Daily Living Skills $\mathrm{F}(4,59)=4.2, \mathrm{p}=.004$, Wilks $\mathrm{l}=$ .22 ; Communication $\mathrm{F}(4,59)=4.8, \mathrm{p}=.002$, Wilks $\mathrm{l}=.25$; Socialisation $\mathrm{F}(4,59)=$ 8.2, $\mathrm{p}<.001$, Wilks $\mathrm{l}=.36$ ). Box's test of equality of covariance matrices was nonsignificant for all three domains and Levene's test was non-significant for all subscales other than the Uncertainty subscale within the Socialisation domain. Separate analyses of variance (ANOVA) were conducted to determine which 
subscales contributed to the significant main effect. Level of ability was entered as the independent variable for each ANOVA with the ASC-ASD subscales of Performance anxiety, Anxious Arousal, Separation Anxiety and Uncertainty entered as the dependent variables, individually. The results are presented in Table 3. Univariate analyses of variance indicated that the Performance Anxiety subscale was found to be significantly higher in those with higher scores on the Communication domain of the VABS-2 and the Anxious arousal subscale was found to be significantly higher in those with lower scores on the Socialisation domain of the VABS-2. Although there was no significant difference between the subscale scores between those with higher and lower scores on the Daily Living Skills domain of the VABS-2, there was a large effect size (.64) for the Performance Anxiety domain, suggesting a lack of power in the current sample size to detect differences. 
Table 3: Per item mean scores on the ASC-ASD anxiety subscales for children with VABS-2 domain scores above and below 70

\begin{tabular}{|c|c|c|c|c|c|c|c|c|c|}
\hline \multirow[b]{2}{*}{$\begin{array}{l}\text { ASC-ASD Subscale Mean } \\
\text { score per item }\end{array}$} & \multicolumn{3}{|c|}{ VABS-2 Daily Living Skills } & \multicolumn{3}{|c|}{ VABS-2 Communication } & \multicolumn{3}{|c|}{ VABS-2 Socialisation } \\
\hline & $\leq 70$ & $\geq 71$ & $\begin{array}{l}\text { ANOVA statistic } \\
\text { Effect size }\end{array}$ & $\leq 70$ & $\geq 71$ & $\begin{array}{l}\text { ANOVA statistic } \\
\text { Effect size }\end{array}$ & $\leq 70$ & $\geq 71$ & $\begin{array}{ll}\text { ANOVA } & \text { statistic } \\
\text { Effect size } & \end{array}$ \\
\hline Performance Anxiety & $\begin{array}{l}.34 \\
(.62)\end{array}$ & $\begin{array}{l}.80 \\
(.73)\end{array}$ & $\begin{array}{l}F(1,62)=5.5 \\
p=.02 \\
d=0.66\end{array}$ & $\begin{array}{l}.23 \\
(.66)\end{array}$ & $\begin{array}{l}.79 \\
(.71)\end{array}$ & $\begin{array}{l}F(1,62)=6.6 \\
p=.01 \\
d=0.80\end{array}$ & $\begin{array}{l}.33 \\
(.65)\end{array}$ & $\begin{array}{l}.78 \\
(.73)\end{array}$ & $\begin{array}{l}F(1,62)=4.5, p=.04 \\
d=0.64\end{array}$ \\
\hline Separation Anxiety & $\begin{array}{l}.80 \\
(.81)\end{array}$ & $\begin{array}{l}.90 \\
(.81)\end{array}$ & $\begin{array}{l}F(1,62)=.20 \\
p=.66 \\
d=0.13\end{array}$ & $\begin{array}{l}.60 \\
(.75)\end{array}$ & $\begin{array}{l}.94 \\
(.81)\end{array}$ & $\begin{array}{l}F(1,62)=1.9 \\
p=.17 \\
d=0.43\end{array}$ & $\begin{array}{l}.72 \\
(.74)\end{array}$ & $\begin{array}{l}.92 \\
(.82)\end{array}$ & $\begin{array}{l}F(1,62)=.70, p=.41 \\
d=0.25\end{array}$ \\
\hline
\end{tabular}




\section{Discussion}

There was variability in parent report of the presence and frequency of a range of anxiety-related symptoms in their autistic children aged 5-6 years as measured by items on the ASC-ASD. However, only two parents reported the absence of symptoms (scoring 0 on all items) while no parent reported all symptoms to be always present (a score of 3). The mean total score was 22.82 and in the small subgroup of participants where parents had disclosed an anxiety diagnosis on an open question, the mean total score was 29.78. The mean of the large sample of older children (age 8-15) in Rodgers et al. (2016b) was 26.19 although this sample contained an unknown proportion of individuals with and without anxiety diagnoses and only children who were able to consent, limiting the extent to which any comparisons can be made between these scores.

Of the four subscales comprising the ASC-ASD, the subscale of 'uncertainty' appeared to be particularly elevated in this group of children. Item 16 in this subscale "My child always needs to be prepared before things happen" received the highest score among the 24 items on the measure. Furthermore, in contrast to other subscales where between 12 and 28 parents reported an absence of symptoms, only two parents reported an absence of symptoms on the 'uncertainty' subscale. These findings warrant discussion in light of the recent focus on intolerance of uncertainty as a possible contributing factor to anxiety in autism and one that is distinguishable from, but related to features of autism (Hodgson et al., 2017). Drawing together the previous evidence and discussion around intolerance of uncertainty, Rodgers et al. (2016a) conclude that intolerance of uncertainty 
is an important mechanism in the both the development and maintenance of anxiety in young children with autism. Given that researchers are now developing interventions which aim to reduce this intolerance of uncertainty in order to reduce anxiety (e.g. Rodgers et al., 2016b), the finding of elevated levels of difficulties with uncertainty in a young population highlights the need for further research into typical and "atypical" levels of difficulties with uncertainty within this age group. Longitudinal studies should also be conducted to identify any predictive power of items or subscales for later diagnosis of an anxiety disorder.

The 'separation anxiety' subscale differentiated between worrying about immediate events (e.g., "my child worries about being away from me"/"my child worries in bed at night because he does not like to be away from me' rated as never by $25-35 \%$ of parents) and future events (e.g. "my child worries that something will happen to someone in the family"/"my child worries that something bad will happen to him/her" both rated as never by $60-70 \%$ of parents). The immediate events in this questionnaire related to separation fears commonly reported in young children (Spence et al., 2001). These may well be related to the child's development level as they have been found to diminish in frequency with age (Weems, 2008). In contrast, future events involved conceptualising a future scenario. This may be particularly difficult for autistic children and adults who have been found less able than their typically developing peers to imagine future events (Lind and Bowler, 2010; Terrett et al., 2013). Further research is needed to better understand possible associations between characteristics of autism and anxiety related to worries about future events. 
Overall, the lowest scores were reported on items describing physiological symptoms related to anxiety (e.g. feel dizzy/faint rated never by 93.2\%; tremble/shake rated never by 75\%; or feels so anxious can’t breathe rated never by 79\%). It could be suggested that many of these items overlap with symptoms of panic disorder, which meta-analyses suggest as the only anxiety diagnosis which is less than twice as prevalent in children with autism (Costello et al., 2005; van Steensel et al., 2011). To report physiological symptoms, parents would need to recognise the physical symptoms in their child and attribute these to anxiety-provoking situations. Children themselves are unlikely to have these abilities at the age of 5 or 6 years (Muris et al., 2010) and it may therefore be difficult for parents to identify these symptoms in their children. Even among school-age autistic children, there has been limited reporting of physiological symptoms by parents (Ozsivadjian et al., 2014; Hallett et al., 2013). Further research is needed to determine whether physiological factors are less commonly experienced by anxious children with autism or whether this is due to a limited capacity to identify and communicate these symptoms.

Previous research has found associations between levels of anxiety and certain child characteristics. For example, Hallett et al. (2013) found higher scores on the VABS were associated with higher levels of anxiety for autistic children aged 4-17 years. In the current study, more advanced communication skills were related to higher levels of performance anxiety. Although the VABS-2 Daily Living Skills and Socialization subscales did not significantly correlate with the performance anxiety score, there was a large effect size, suggesting this requires further investigation. Spence et al. (2001) examined anxiety symptoms in children with typical development aged 2.5-6.5 years and 
found performance anxiety items were rated highly for 4-5 year olds but not younger children in the sample, which they suggest may be due to developing cognitive factors relating to self-consciousness. The interaction between developing language skills and self-consciousness (e.g. Rochat, 2003) may also be influencing the relationship between higher levels of performance anxiety in those with more advanced communication scores on the VABS-2 noted within this sample; something that would benefit from further exploration with self-report studies which include measures of self-consciousness. Longitudinal research examining developmental trajectories and patterns of anxiety and anxiety-related symptomatology could also make an important contribution to our knowledge in this area.

\section{Limitations}

It is important to exercise caution when interpreting the results from this study, as the ASC-ASD has not yet been validated with children younger than 8 years. In addition, while the psychometric properties of this measure are promising, further validation is warranted. However, in the absence of measures better suited to the task, the ASC-ASD has provided a means of exploring and describing anxiety-related symptoms in this population, something that has been previously overlooked.

This study relied on parent report and there is a risk that parent perceptions of anxiety symptomatology differ from those of their child. There has, however, been little research involving self-report in young children and studies comparing parent and selfreport of anxiety-related symptoms have generally involved children older than those in the current study. For example, Bitsika and Sharpley (2015) compared responses of 140 
autistic boys aged 6-18 years (mean age 11.2 years) and their parents using the Child and Adolescent Symptom Inventory (CASI). Parents in this study rated their sons significantly higher on general anxiety disorder and social phobia than did their child. There were, however, non-significant differences between parents and their sons on 5 of the remaining 6 anxiety disorders. Further investigations are warranted to determine if children under the age of 6 years are able to successfully complete self-report measures of anxiety and how their responses compare to those of their parents. The use of biological indicators of anxiety such as cortisol in this age group may also provide another area for further investigation. While the association between cortisol and anxiety in autistic children is not well established, particularly in the target age group for this study, some research has shown a significant association between cortisol and generalized anxiety disorder in autistic children (Bitsika et al., 2015).

This study also measures anxiety-related symptomatology at one point in time and longitudinal research is needed to improve our knowledge of how anxiety-related symptoms for autistic children may change over time and whether a specific profile of anxiety-related symptoms at this young age is predictive of later diagnosis. There is evidence that age may be a factor in the severity and type of anxiety-related symptoms experienced by autistic children and improving our knowledge of these trajectories will inform treatment.

\section{Conclusion}

This study provides preliminary findings regarding anxiety-related symptomatology reported by parents of young autistic children using a newly published measure of 
anxiety developed specifically for autistic children aged 8-16 years. It is evident that the 5-6 year olds in this study experience a range of anxiety-related symptoms according to this measure, with the most prevalent symptoms relating to uncertainty. Early interventions that focus specifically on ameliorating these symptoms of anxiety may therefore prove particularly effective for this population. Research is needed to identify effective early intervention strategies and to determine if such interventions can protect against the development of anxiety disorder in later childhood, adolescence and adulthood. 


\section{References}

Beesdo K, Knappe S and Pine D. (2009) Anxiety and anxiety disorders in children and adolescents: Developmental issues and implications for DSM-V. Psychiatric Clinics of North America 32: 483-524.

Biedel D and Turner S. (2005) Childhood anxiety disorders: A guide to research and treatment, New York, NY: Routledge.

Birmaher B, Brent D, Chiapetta L, et al. (1999) Psychometric properties of the screen for child anxiety related emotional disorders (SCARED): A replication study. Journal of the American Academy of Child \& Adolescent Psychiatry 38: 1230-1236.

Bitsika V and Sharpley C. (2015) Variation in the profile of anxiety disorders in boys with an ASD according to method and source of assessment. Journal of Autism \& Developmental Disorders 45: 1825-1835.

Bitsika V, Sharpley CF, Andronicos NM, et al. (2015) A test of the 'parent distortion’ hypothesis when assessing generalised anxiety disorder in boys with an autism spectrum disorder. Research in Autism Spectrum Disorders 15: 42-52.

Costello EJ, Egger H and Angold A. (2005) The developmental epidemiology of anxiety disorders: Phenomenology, prevalence, and comorbidity. Child and Adolescent Psychiatric Clinics of North America 14: 631-648.

Davis T, Hess J, Moree B, et al. (2011) Anxiety symptoms across the lifespan in people diagnosed with autistic disorder. Research in Autism Spectrum Disorders 5: 112118. 
Eaves L, Wingert H, Ho H, et al. (2006) Screening for autism spectrum disorders with the social communication questionnaire. Developmental and Behavioral Pediatrics 27: 96-103.

Franz L, Angold A, Copeland W, et al. (2013) Preschool anxiety disorders in pediatric primary care: Prevalence and comorbidity. Journal of the American Academy of Child \& Adolescent Psychiatry 52: 1294-1303.

Gadow K, DeVincent C, Pomeroy J, et al. (2004) Psychiatric symptoms in preschool children with PDD and clinic and comoparison samples. Journal of Autism \& Developmental Disorders 34: 379-393.

Gadow K and Sparafkin J. (1997) Early Childhood Symptom Inventory-4 norms manual, Stony Brook, NY: Checkmate Plus.

Gadow K and Sparafkin J. (2000) Early Childhood Symptom Inventory-4 screening manual, Stony Brook, NY: Checkmate Plus.

Hallett V, Lecavalier L, Sukhodolsky DG, et al. (2013) Exploring the manifestations of anxiety in children with autism spectrum disorders. Journal of Autism and Developmental Disorders 43: 2341-2352.

Hodgson AR, Freeston MH, Honey E, et al. (2017) Facing the unknown: Intolerance of uncertainty in children with autism spectrum disorder. Journal of Applied Research in Intellectual Disabilities 30: 336-344.

Kerns CM, Kendall PC, Berry L, et al. (2014) Traditional and atypical presentations of anxiety in youth with autism spectrum disorder. Journal of Autism and Developmental Disorders 44: 2851-2861. 
Lind SE and Bowler DM. (2010) Episodic memory and episodic future thinking in adults with autism. Journal of abnormal psychology 119: 896-905.

Magiati I, Ong C, Lim X, et al. (2015) Anxiety symptoms in young people with autism spectrum disorder attending special schools: Association with gender, adaptive functioning and autism symptomatology. Autism 20: 306-320.

Muris P, Mayer B, Freher NK, et al. (2010) Children's internal attributions of anxietyrelated physical symptoms: Age-related patterns and the role of cognitive development and anxiety sensitivity. Child Psychiatry \& Human Development 41: 535-548.

Ozsivadjian A, Hibberd C and Hollocks MJ. (2014) Brief report: The use of self-report measures in young people with autism spectrum disorder to access symptoms of anxiety, depression and negative thoughts. Journal of Autism and Developmental Disorders 44: 969-974.

Rapee RM. (2013) The preventative effects of a brief, early intervention for preschool aged children at risk for internalising: follow - up into middle adolescence. Journal of Child Psychology and Psychiatry 54: 780-788.

Roberts J, Adams D, Heussler H, et al. (in press) Protocol for a prospective longitudinal study investigating the participation and educational trajectories of Australian students with autism. BMJ Open.

Rochat P. (2003) Five levels of self-awareness as they unfold early in life. Consciousness and Cognition 12: 717-731. 
Rodgers J, Hodgson A, Shields K, et al. (2016a) Towards a treatment for intolerance of uncertainty in young people with autism spectrum disorder: Development of the Coping with Uncertainty in Everyday Situations (CUESC) programme. Journal of Autism and Developmental Disorders.

Rodgers J, Wigham S, McConachie H, et al. (2016b) Development of the anxiety scale for children with autism spectrum disorder (ASC - ASD). Autism Research 9:

1205-1215.

Rutter M, Bailey A and Lord C. (2007) Social Communication Questionnaire, Los Angeles, CA: Western Psychological Services.

Seo T, Kanda T and Fujikoshi Y. (1995) The effects of nonnormality on tests for dimensionality in canonical correlations and MANOVA Models. Journal of Multivariate Analysis 52: 325-337.

Sparrow S, Dominic V, Cicchetti D, et al. (2005) Vineland Adaptive Behavior Scales, Circle Pines, MN: American Guidance Service.

Spence SH, Rapee R, McDonald C, et al. (2001) The structure of anxiety symptoms among preschoolers. Behaviour research and therapy 39: 1293-1316.

Tabachnick B and Fidell L. (2007) Using multivariate statistics, Boston, MA: Pearson. Terrett G, Rendell PG, Raponi-Saunders S, et al. (2013) Episodic future thinking in children with autism spectrum disorder. Journal of Autism and Developmental Disorders 43: 2558-2568. 
van Steensel F, Bögels S and Perrin S. (2011) Anxiety disorders in children and adolescents with autistic spectrum disorders: A meta-analysis. Clinical Child and Family Psychology Review 14: 302.

Weems CF. (2008) Developmental trajectories of childhood anxiety: Identifying continuity and change in anxious emotion. Developmental Review 28: 488-502.

White SW, Oswald D, Ollendick T, et al. (2009) Anxiety in children and adolescents with autism spectrum disorders. Clinical Psychology Review 29: 216-229.

Wigham S and McConachie H. (2014) Systematic review of the properties of tools used to measure outcomes in anxiety intervention studies for children with autism spectrum disorders. PLoS ONE 9: e85268. 